\title{
Inhibition of tissue factor pathway inhibitor (TFPI) by ARC19499 improves clotting of hemophiliac blood
}

\author{
Monika E Gorczyca ${ }^{1}$, Bernd Jilma* ${ }^{*}$, Christoph Male², Sylvia Reitter ${ }^{3}$, James C Gilbert ${ }^{4}$, Ingrid Pabinger ${ }^{3}$ \\ From 16th Scientific Symposium of the Austrian Pharmacological Society (APHAR) \\ Vienna, Austria. 25-27 November 2010
}

\section{Background}

Hemophilia A and B are disorders resulting from a deficiency in factor VIII (FVIII), and factor IX (FIX), respectively. Tissue Factor (TF) is a key component of the extrinsic pathway and plays a role in the coagulation defect of hemophilic blood. Neutralizing the activity of TFPI represents a promising treatment concept in patients with hereditary or acquired hemophilia. ARC19499 is a polyethylene glycol (PEG)-conjugated aptamer that binds to TFPI and inhibits its function as a negative regulator of coagulation.

\section{Methods}

We evaluated the effect of the anti-TFPI aptamer ARC19499 on thrombin generation and clot formation in 40 hemophilia patients (congenital hemophilia: 29 adults, 10 children, one acquired hemophilia patient) and 27 healthy male controls. Concentration-effect curves of ARC19499 were assessed by the calibrated automated thrombogram (CAT) and rotational thrombelastometry (ROTEM) with and without corn trypsin inhibitor (CTI) in whole blood and platelet-poor plasma.

\section{Results}

Clotting patterns were significantly compromised in patients vs. controls. Measured FVIII:C levels in hemophiliacs correlated with parameters of the CAT assay (except lag time) and also with the clotting time assessed by ROTEM. ARC19499 had a concentrationdependent pro-hemostatic effect in CAT and ROTEM. ROTEM results with and without CTI were consistent.

\footnotetext{
* Correspondence: bernd.jilma@meduniwien.ac.at

'Department of Clinical Pharmacology, Medical University of Vienna, 1090 Vienna, Austria

Full list of author information is available at the end of the article
}

ARC19499 normalized ROTEM clotting parameters and rendered CAT patterns of hemophilia patients practically indistinguishable from those of controls. In patients, ARC19499 normalized the endogenous thrombin potential, time-to-peak and the start tail, and increased peak thrombin more than 2-fold. ARC19499 raised peak thrombin in patients with FVIII:C under 1\% to levels within the range of healthy controls and higher than baseline in patients with FVIII:C above 5\%. Effective concentrations of ARC19499 started at $2 \mathrm{nM}$. In an acquired hemophilia patient ARC19499 worked synergistically with activated prothrombin-complex concentrate/factor VIII inhibitor bypass activity (FEIBA) pre-treatment. Similarly, in blood spiked with a FVIII antibody, simulating a state of acquired hemophilia, ARC19499 restored normal coagulation.

\section{Conclusions}

The anti-TFPI aptamer ARC19499 effectively enhanced coagulation in blood of patients with congenital or acquired hemophilia and therefore deserves further evaluation in clinical trials.

\section{Author details}

1Department of Clinical Pharmacology, Medical University of Vienna, 1090 Vienna, Austria. ${ }^{2}$ Department of Pediatrics and Adolescent Medicine, Medical University of Vienna, 1090 Vienna, Austria. ${ }^{3}$ Department of Medicine I, Division of Hematology and Hemostasis, Medical University of Vienna, 1090 Vienna, Austria. ${ }^{4}$ Archemix Corp., Cambridge, MA 02142, USA.

Published: 16 November 2010

doi:10.1186/1471-2210-10-S1-A44

Cite this article as: Gorczyca et al:: Inhibition of tissue factor pathway inhibitor (TFPI) by ARC19499 improves clotting of hemophiliac blood. BMC Pharmacology 2010 10(Suppl 1):A44. 